\title{
UNSTEADY HYDROMAGNETIC COUETTE FLOW UNDER AN OSCILLATING PRESSURE GRADIENT AND UNIFORM SUCTION AND INJECTION
}

\author{
Jennilee Veronique ${ }^{1}$, Sreedhara Rao Gunakala ${ }^{2 *}$, Victor M. Job ${ }^{3}$ \\ ${ }^{1,2,3}$ Faculty of Science and Technology, The University of the West Indies, Trinidad \\ ${ }^{1}$ Email: jennilee.veronique@my.uwi.edu \\ ${ }^{2}$ Email: Sreedhara.rao@sta.uwi.edu*(Corresponding author) \\ ${ }^{3}$ Email: victor.job@sta.uwi.edu (Corresponding author)
}

\begin{abstract}
In this paper, we consider the unsteady hydromagnetic Couette flow of a viscous incompressible flow between two infinitely-long horizontal parallel plates under an oscillating pressure gradient. We apply a constant magnetic field which is perpendicular to the plates, and there is uniform suction and injection through the plates. The governing equation for fluid motion within the channel is discretized with the help of the Galerkin Finite Element Method. The effects of the Suction parameter $S$, Hartmann number $\mathrm{Ha}$, Reynolds number $R e$, the amplitude of the pressure gradient $k$ and the oscillation frequency of the pressure gradient $\omega$ on the velocity distribution are investigated.
\end{abstract}

Keywords: Couette Flow; Finite Element Analysis; Hydromagnetics; Oscillatory Flow; Flow with Suction and Injection.

https://doi.org/10.47412/JCLZ2920

\section{Introduction}

The study of unsteady Couette flow under varying different physical effects, such as uniform suction and injection, an externally-applied magnetic field and an oscillating pressure gradient, has been studied extensively in the last few decades. This type of fluid flow has been mathematically examined in a number of different ways such as experimentation [1], finite difference method [2-4], finite element method [5,6], perturbation technique [7], Laplace transformation technique [8-9] and network numerical simulation [10].

Hydromagnetics, also known as magnetohydrodynamics (MHD), focuses on the dynamics of magnetic fields in electrically conducting fluids such as in liquid metals and plasma [11]. Hydromagnetics combines the principles of both fluid dynamics and electromagnetism. The study of Couette flow is applicable to MHD power generators, rotating hydromagnetic generators and other centrifugal machines in a rotating system [3]. In addition, it also holds interest in many areas of industrial engineering designs [7]. Suction or injection on the boundary contributes significantly in the field of aerodynamics and space sciences [12]. Its main purpose is to influence the flow of fluid in the channel.

MHD Couette flow has been an area of interest and widely researched. Willis and Barenghi [1] studied rotating hydromagnetic Couette flow using a comparison between numerical and experimental methods. This study seeks to examine the effects which have been induced on Couette flow by a magnetic field which is applied externally. It was found that the magnetic field increases the velocity regardless of the temporal extent of movement and the rotation speed.

The flow of fluids under the effects of suction and injection has been investigated by researchers due to its extensive applications in engineering and industry. Uwanta and Hamza [12] studied the effects of suction 
and injection on unsteady MHD convective flow of reactive viscous fluid between vertical porous plates with thermal diffusion. This study showed that suction and injection, thermal diffusion, reaction consumption and thermal and solutal buoyancy are instrumental in directing the transport phenomena. A similar investigation was conducted by Jha, Aina, Ajiya [13] in their investigation into the role of suction and injection on MHD natural convection flow in a vertical micro channel. In this case, it was observed that there has been marked significance when the ambient wall temperature difference ratio decreases with the effects of suction and injection parameter on the temperature jump and the micro-channel velocity slip. In addition, the effects of suction and injection on the unsteady flow between two parallel plates with variable properties were examined by Attia [14]. It was found that the effects of the suction on velocity are dependent highly on the viscosity parameter. The study of hydromagnetic Couette flow due to ramped motion of one of the porous plates [15] is also examined. For their study, observations were made that the existence of the injection parameter causes the velocity to increase and with the suction parameter, the velocity decreases at the moving porous plate. This occurs regardless of whether there is fixed magnetic lines of force for the fluid or the moving porous plate. An analysis of unsteady MHD Couette flow and heat transfer in a rotating horizontal channel with injection/ suction by Gupta and Jain [7] was discussed and used an analytical approach by applying perturbation technique in obtaining its solution. The study revealed that there has been considerable impact on the hydromagnetic flow and heat transfer because of the combined effects of the rotation parameter, the magnetic field, thermal slip, injection/suction, permeability, heat generation/absorption and Prandtl number. In a similar manner, Attia [2] focused on the unsteady MHD Couette flow with heat transfer in the presence of uniform suction and injection, however, this study applied a method of finite differences in obtaining its solution. The study revealed that there was a prominent effect on the velocity and temperature distributions due to the magnetic field and suction or injection velocity. Also, when the magnetic field is increased, the temperature also increases when the time is small and when the time is large, the temperature decreases when the magnetic field is increased.

The effects of oscillatory flows have been an area of interest and have been studied by several researchers. Ahmed, Sheikh and Barua [16] examined the effect of fluid suction on an oscillatory MHD channel flow with heat transfer. The investigation looked at two infinite parallel porous walls and assumed that the pressure gradient was oscillatory in nature. It was discovered that the magnetic field heighten the rates of heat transfer at the walls and temperature is decreased due to the fluid suction. Fluid suction also assists in the lamina flow control. Singh [9] examined suction/injection effects on MHD oscillatory convective flow in a rotating vertical porous channel with thermal radiation. The author showed that velocity decreases when the rotation of the channel is increasing. The velocity also decreases when the radiation parameter, the frequency of oscillation and the Prandtl number increases. Job and Gunakala [6] examined unsteady MHD free convection Couette flow between two vertical permeable plates using a finite element analysis approach. The study explored a fluid existing between two infinite permeable plates in the company of thermal radiation where an exponentially decaying pressure gradient is present. The results indicated that there was a reduction in the velocity and temperature when the suction parameter increases, and an increase in fluid temperature when the magnetic parameter increases.

The finite element method is a powerful and effective numerical method which is applied to real world problems including heat transfer, solid and fluid mechanics and electrostatic problems with complicated geometry. In applying this method, the domain given is viewed as a collection of subdomains and over each subdomain, the governing equation is approximated using any of the traditional variational methods. In the present study, the Galerkin finite element method is used to investigate unsteady hydromagnetic Couette flow under a sinusoidally-oscillating pressure gradient and uniform suction and injection.

\section{Description of the Problem}

The flow of a viscous incompressible flow between two infinitely long horizontal parallel plates is considered. In the $x$ and $z$ directions, these two plates are infinite and are stationed at $y=-h$ and $y=h$. 
The lower plate is stationary while the upper plate moves with the constant velocity $U_{0}$. The fluid flows with uniform injection through the lower plate and uniform suction through the upper plate. The fluid flows between the two plates are influenced by an oscillating pressure gradient $\frac{\partial p}{\partial x}$ in the $x$-direction, a uniform suction from above and injection from below which are applied at $t=0$. A uniform applied constant magnetic field $B_{0}$, perpendicular to the plates in the positive $y$-direction is applied to the whole system.

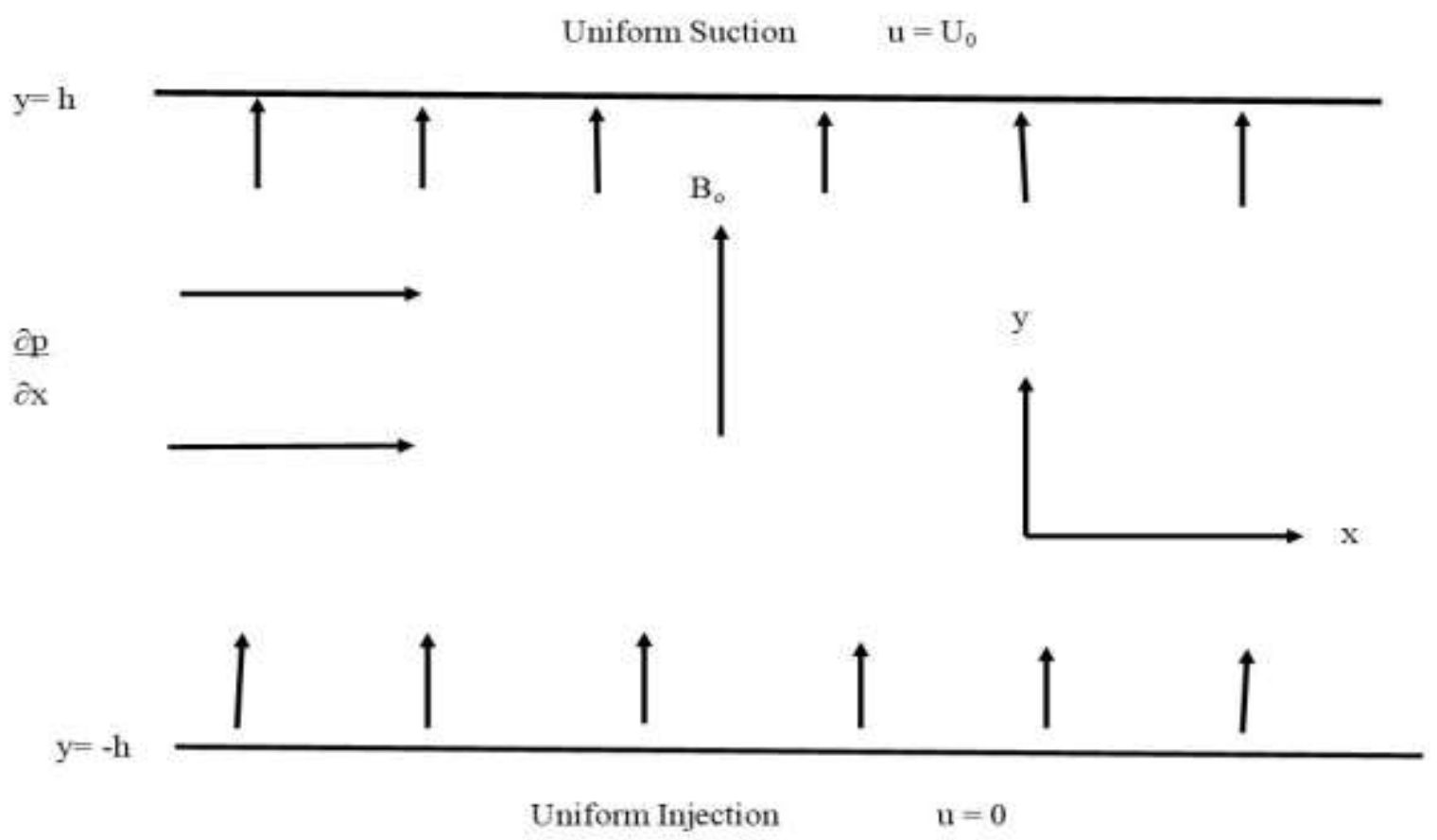

Figure 9: Schematic Diagram of the Physical System

According to the above description of the problem, we made the following assumptions:

1. The flow is incompressible; that is, $\rho$ is a constant.

2. There is a very small magnetic Reynolds number.

3. The flow examined is laminar, fully developed and oscillatory.

4. The pressure gradient in the channel undergoes sinusoidal oscillations.

Since the suction/injection through the plates is uniform, the velocity vector of the fluid is written as $\vec{u}(y, t)=u(y, t) \vec{\imath}+v_{0} \vec{\jmath}$. From the above assumptions, the fluid flow is governed by the following momentum equation:

$$
\rho \frac{\partial u}{\partial t}+\rho v_{0} \frac{\partial u}{\partial y}=-\frac{\partial p}{\partial x}+\mu \frac{\partial^{2} u}{\partial y^{2}}-\sigma B_{0}^{2} u
$$

where $\rho, \mu$ and $\sigma$ are the density, viscosity and electrical conductivity of the fluid respectively. 
The initial condition is

$$
u=0 \text { at } t=0
$$

and the boundary conditions are

$$
\begin{aligned}
& u=0 \text { at } y=-h \\
& u=U_{0} \text { at } y=h
\end{aligned}
$$

Equations (1)-(4) are transformed in dimensionless form by using the non-dimensional variables defined as follows:

$$
\hat{x}=\frac{x}{h}, \hat{y}=\frac{y}{h}, \hat{u}=\frac{u}{U_{0}}, \hat{p}=\frac{p}{\rho U_{0}^{2}}, \hat{t}=\frac{t U_{0}}{h}
$$

where $S=\frac{v_{0}}{U_{0}}$ is the suction parameter, $H a=B_{0} h \sqrt{\frac{\sigma}{\mu}}$ is the Hartmann number and $R e=\frac{\rho h U_{0}}{\mu}$ is the Reynolds number. On dropping all hats and taking the non-dimensional pressure gradient to be

$$
\frac{\partial p}{\partial x}=-k \cos (\omega t)
$$

with dimensionless amplitude $k$ and oscillation frequency (Strouhal number) $\omega$ of the pressure gradient, we get the non-dimensional equation

$$
\frac{\partial u}{\partial t}+S \frac{\partial u}{\partial y}=k \cos (\omega t)+\frac{1}{R e} \frac{\partial^{2} u}{\partial y^{2}}-\frac{H a^{2}}{R e} u
$$

The dimensionless initial and boundary conditions are

$$
\begin{aligned}
& u=0 \text { at } t=0 \\
& u=0 \text { at } y=-1 \\
& u=1 \text { at } y=1
\end{aligned}
$$

\section{Numerical Solution of the Problem}

The equation is solved numerically using Galerkin's finite element method under the initial and boundary conditions to determine the velocity distributions for different values of the parameters $\mathrm{Ha}, \mathrm{Re}, \mathrm{S}, \mathrm{k}$ and $\omega$. According to the Galerkin's finite element method [17], a spatial semi-discretization of the problem was performed such that the associated trial and test function spaces are equal, and the finite element approximation is represented using piecewise quadratic Lagrange interpolation functions. The CrankNicholson scheme was then applied to obtain a system of linear equations, which is solved using the mathematical software MATLAB. 


\section{Discussion of Results}

The approximate solutions for the velocity distribution was calculated for various values of time, Hartmann number, Reynolds number, suction parameter, amplitude of the pressure gradient and the frequency of oscillation. The values time $t=0.5,1,2$; Hartmann number $H a=0,1,3$; Reynolds number $R e=$ $0.5,1,1.5$; Suction parameter $S=0,1,2$; oscillation frequency $\omega=0,0.5,1$ and oscillation amplitude $k=$ $0,1,3,5,7,9$ were used in analysing the numerical results. The Reynolds number $R e=1$ and the parameters $k$ and $\omega$ are taken to be 5 and 0 respectively throughout the analysis unless otherwise stated.

Fig. 2 displays the velocity distribution for various values of the time, beginning from $t=0$ and is examined at $S=1, R e=1$ and $H a=1$. It can be seen that as time increases, the velocity $u$ also increases. The flow velocity approaches a steady state with increasing $t$. This result is in agreement with the result obtained by Attia [2] in the study of the unsteady MHD Couette flow with heat transfer in the presence of uniform suction and injection.

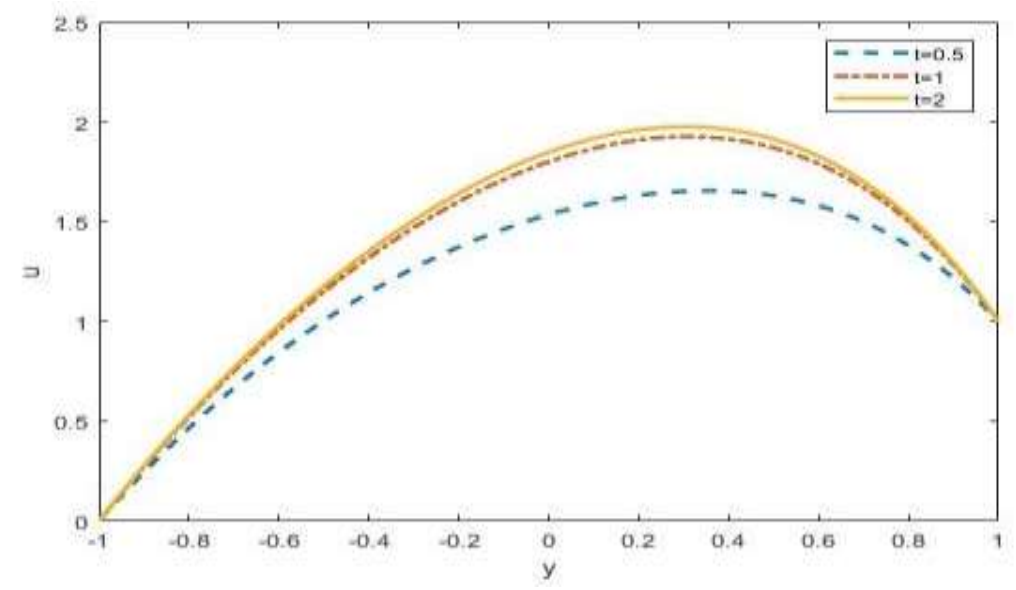

Figure 2: Time development of the velocity $u$ with $S=1, R e=1, H a=1, k=5, \omega=0$

Fig. 3 presents the time development of the velocity $u$ and the effects of the Hartmann number $H a$ on the velocity. In this case, the suction parameter, $S=0$, Reynolds number, $R e=1$ and time $t=4$. It can be observed that at its steady state time, the velocity $u$ decreases as the Hartmann number $\mathrm{Ha}$ increases. This occurs because of the magnetic damping force on the velocity $u$ increasing. Attia [2] obtained a similar result in the examination of the unsteady MHD Couette flow with heat transfer in the presence of uniform suction and injection.

The suction parameter effects on the time development of the velocity $u$ is shown in Fig. 4. In this situation, the Hartmann number, $\mathrm{Ha}=0, \mathrm{Re}=1$ and $t=4$. In this figure, it is noticed that as the suction increases, the velocity $u$ decreases. This can be attributed to fluid convection from areas in the lower half of the channel to the centre, in which the speed of the fluid is higher. In the study unsteady MHD Couette flow with heat transfer in the presence of uniform suction and injection conducted by Attia [2] the results obtained concur with the results of this study. 


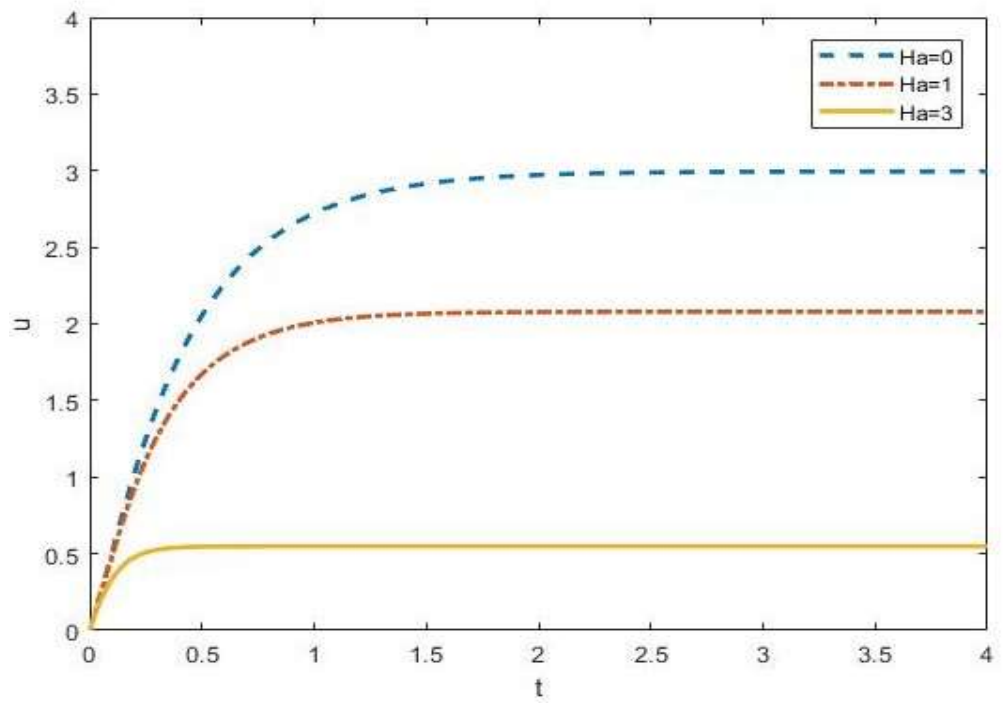

Figure 3: Effect of Ha on the time variation of $u$ with $S=1, R e=1, H a=1, k=5, \omega=0$

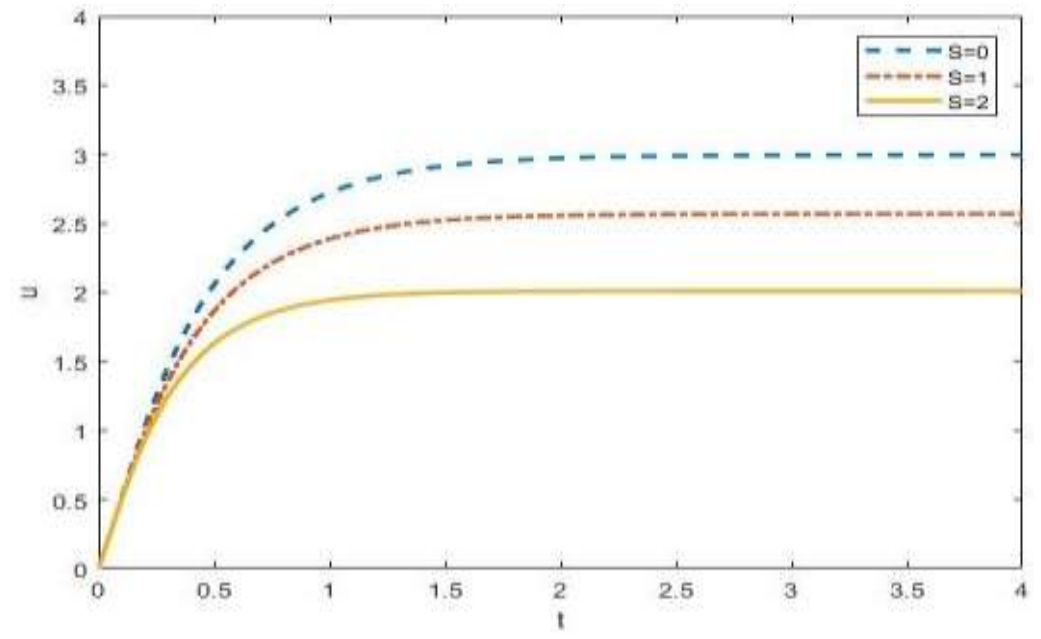

Figure 4: Effect of $\mathrm{S}$ on the time variation of $u$ with $R e=1, H a=1, k=5, \omega=0$

The Reynolds number effects on the time development of the velocity $u$ is displayed in Fig. 5. In this case, the Hartmann number $\mathrm{Ha}=0$, the suction parameter $S=0$ and $t=4$. It is shown in the figure that the velocity also increases as the Reynolds number increases. This means that the flow continues to be smooth with very little turbulence. 


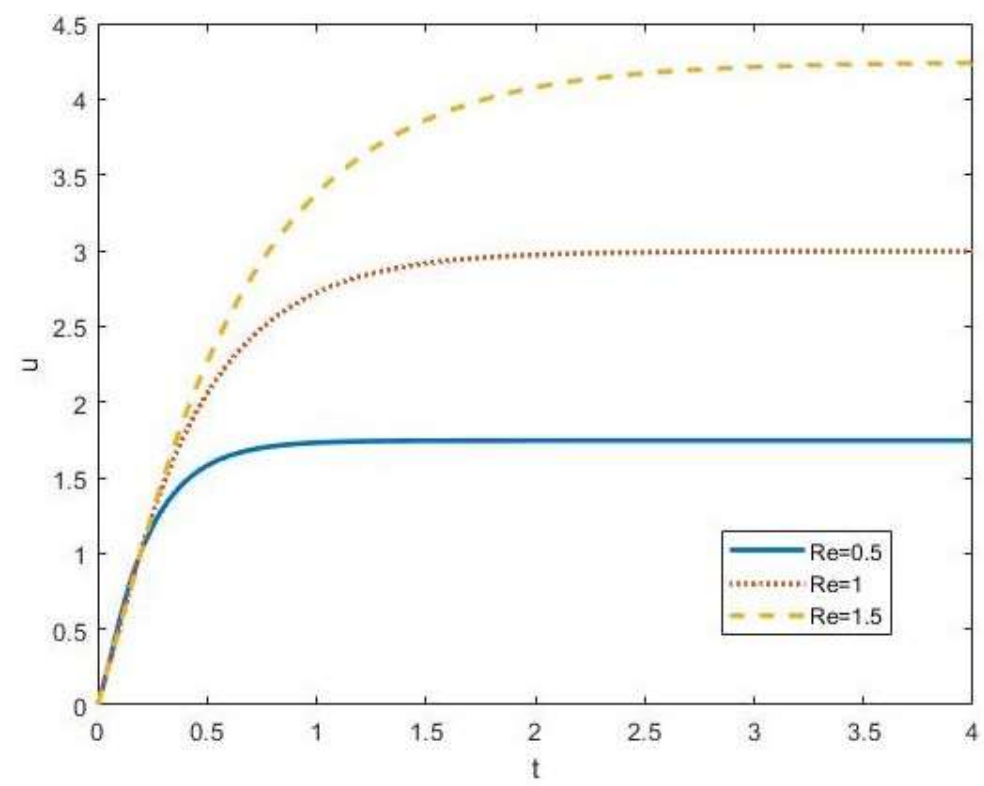

Figure 5: Effect of $\operatorname{Re}$ on the time variation of $u$ with $S=1, H a=1, k=5, \omega=0$

Figs. 6-8 shows the effect of the amplitude of the pressure gradient $k$ on the time development of the velocity of $u$ for various values of the frequency of oscillation $\omega$. In Fig. $6, \omega=0$, the Hartmann number $H a=1$, the suction parameter $S=1$, Reynolds number $R e=1$ and $t=4$ is taken. In this case, as the value of $k$ increases the velocity also increases. In Fig. 7, $\omega=1$, the suction parameter $S=1$, the Hartmann number $H a=1$, Reynolds number $R e=1$ and $t=1.85$. The figure revealed that as the value of $k$ increases the velocity increases faster than at the point where $\omega=0$, before it decreases. This indicates an increase in oscillation throughout the flow. A similar occurrence is shown in Fig. 8 when $\omega=5$. For this situation, $t=0.49$ and we can also observe that the velocity increases as $k$ also increases. The increase occurs rapidly indicating that oscillation is faster as the value of $k$ increases and there exists a free flow at $k=0$, increasing fluid speed.

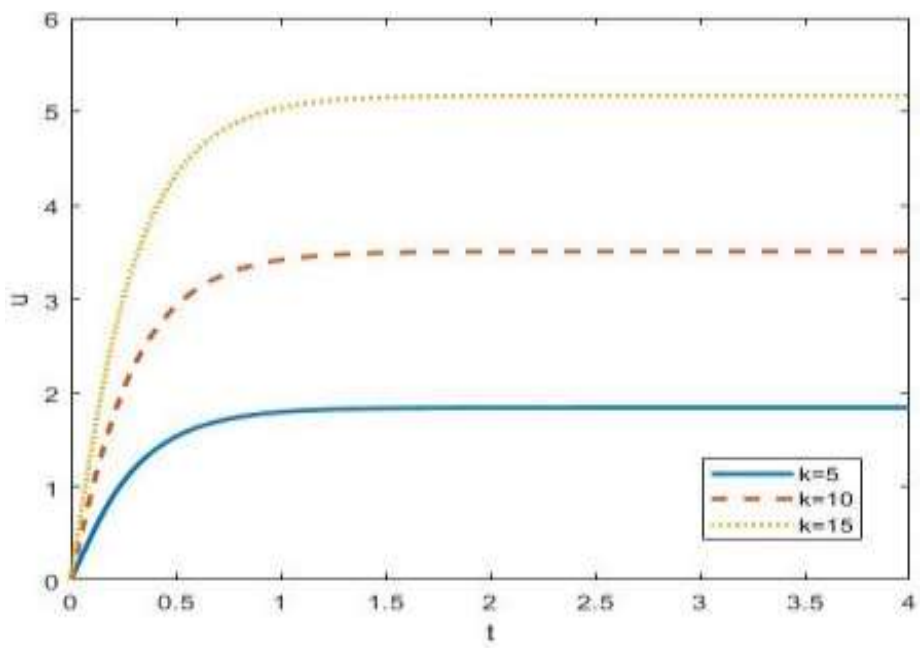

Figure 6: Effect of $\mathrm{k}$ on the time variation of $u$ with $S=1, R e=1, H a=1, \omega=0$ 


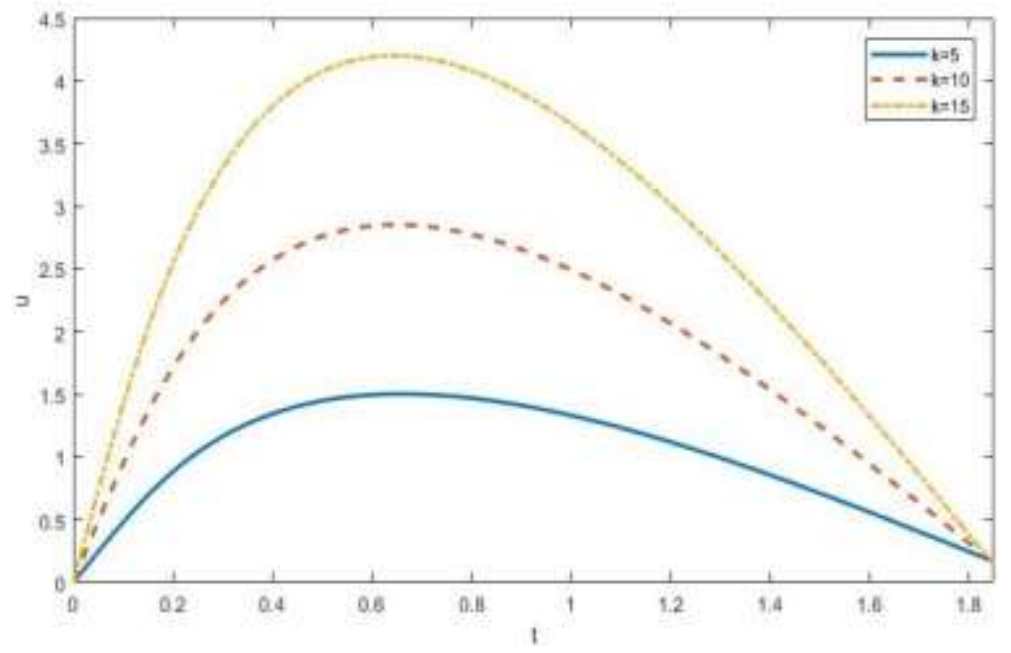

Figure 7: Effect of k on the time variation of $u$ with $S=1, R e=1, H a=1, \omega=1$

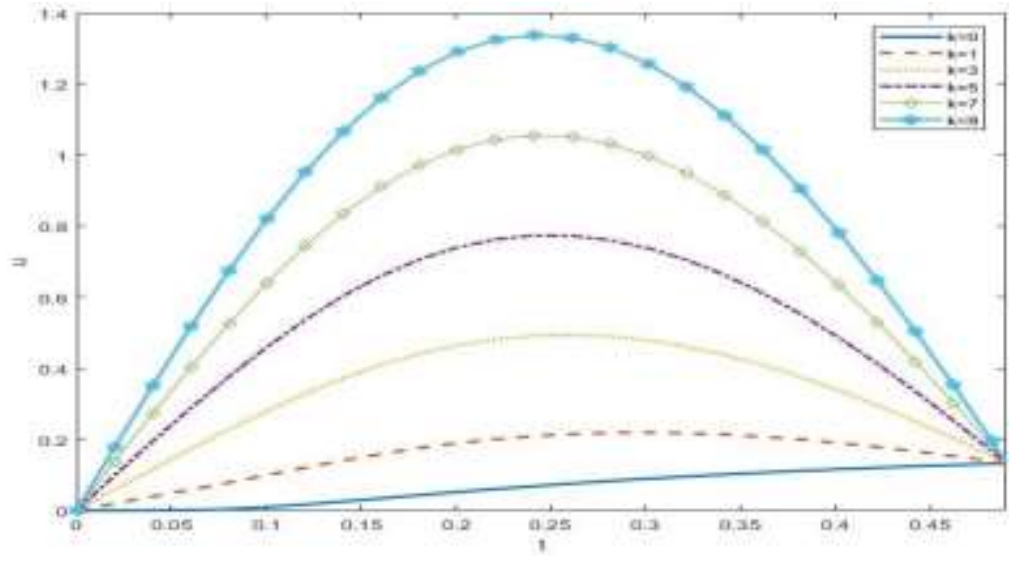

Figure 8: Effect of $\mathrm{k}$ on the time variation of $\boldsymbol{u}$ with $S=1, \operatorname{Re}=1, \mathrm{Ha}=1, k=5, \omega=5$

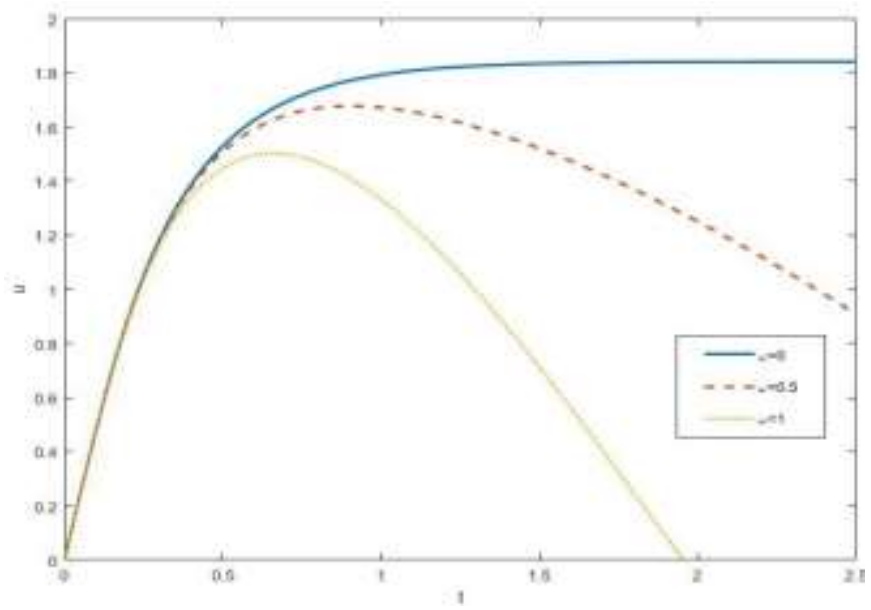

Figure 9: Effect of $\omega$ on the time variation of $u$ with $S=1, R e=1, H a=1, k=5$ 
Fig. 9 displays the effect of $\omega$ on the time development of the velocity $u$. In this case, the suction parameter $S=1$, the Hartmann number $H a=1$ and the Reynolds number $R e=1$. In the figure, it is shown that velocity decreases as $\omega$ increases, therefore pressure is decreasing as the frequency of oscillation increases.

\section{Conclusion}

The unsteady hydromagnetic Couette flow under an oscillating pressure gradient and uniform suction and injection was investigated in this study. The effects of the suction parameter, Hartmann number, Reynolds number, the amplitude of the pressure gradient $k$ and the frequency of oscillation $\omega$ on the velocity of the fluid have been examined. It was revealed that the suction parameter, Hartmann number and Reynolds number has a marked effect on velocity. In the case of $k$ and various values of the frequency of oscillation $\omega$, it was found that velocity increases as the value of $k$ increases. In addition, as the frequency of oscillation increases, the velocity decreases.

\section{References}

[1] Willis, Ashley P, and Carlo F Barenghi. "Hydromagnetic Couette Flow-Numerical Methods and Comparison with Experiment." $12^{\text {th }}$ International Couette-Taylor Workshop, September 6-8, 2011. USA: Evanston, Illinois.

[2] Attia, Hazem Ali. "Unsteady MHD Couette flow with heat transfer in the presence of uniform suction and injection." Mechanics and Mechanical Engineering 12, no. 2 (2008): 165-170.

[3] Chutia, Muhim, Tabendra Nath, and Pranob Jyoti. "Numerical Solution of Unsteady Hydromagnetic Couette Flow in a Rotating System Bounded by Porous Plates with Hall Effects." International Journal of Computer Applications 171 (August 2017): 1-6. doi:10.5120/ijca2017914967.

[4] Kala, Bhim. "Numerical Study of the Effects of Suction and Pressure Gradient on an Unsteady MHD Fluid Flow between Two Parallel Plates in a Non-Darcy Porous Medium." Asian Research Journal of Mathematics 3 (January 2017): 1-14. doi:10.9734/ARJOM/2017/33002.

[5] Rabari, N.S, A.S Gor, and Pravinchandra Bhathawala. (2014). "Finite Element Solution of One Dimensional Flow in Unsaturated Porous Media." IOSR Journal of Mathematics 10(3): 49-57.

[6] Job, Victor M, and S Rao Gunakala. "Unsteady MHD Free Convection Couette Flow between Two Vertical Permeable Plates in the Presence of Thermal Radiation Using Galerkin's Finite Element Method." International Journal of Mechanical Engineering 2, no. 5 (2013): 99-110. 45.

[7] Gupta, VG, and Ajay Jain. "An Analysis of Unsteady MHD Couette Flow and Heat Transfer in a Rotating Horizontal Channel with Injection/Suction." International Journal of Latest Technology in Engineering, Management \& Applied Science 6 (2016): 28-45.

[8] Seth, GS, Md S Ansari, and R Nandkeolyar. "Effects of rotation and magnetic field on unsteady Couette flow in a porous channel," Journal of Applied Fluid Mechanics 4(3): 95-103 (2011).

[9] Singh, K. D. "Injection/Suction Effects on hydromagnetic oscillatory convective flow in a rotating vertical porous channel with thermal radiation.” In Proc. Ind. Natn. Sci. Acad, 79:79-90 (2013).

[10] Bég, O.A., J. Zueco, S.K. Ghosh, and A. Heidari. "Unsteady Magnetohydrodynamic Heat Transfer in a Semi-Infinite Porous Medium with Thermal Radiation Flux: Analytical and Numerical Study." Advances in Numerical Analysis, 2011: 304124 (2011).

[11] Dorch, Søren Bertil F. "Magnetohydrodynamics." Scholarpedia 2, no. 4 (2007): 2295.

[12] Uwanta, IJ, andMMHamza. "Effect of suction/injection on unsteady hydromagnetic convective flow of reactive viscous fluid between vertical porous plates with thermal diffusion." International scholarly research notices 2014 (2014).

[13] Jha, Basant K, Babatunde Aina, and AT Ajiya. "Role of suction/injection on MHD natural convection flow in a vertical microchannel." Int. J. Energy Technol 7, no. 2 (2015): 30-39.

[14] Attia, Hazem Ali. "The effect of suction and injection on the unsteady flow between two parallel plates with variable properties." 8, no. 1 (2005): 17-22. 
[15] Jha, BK, and HM Jibril. "Unsteady hydromagnetic Couette flow due to ramped motion of one of the porous plates." International Journal of Applied Mechanics and Engineering 18, no. 4 (2013): 1039-1056. [16] Ahmed, N, AH Sheikh, and DP Barua. "Effect of Fluid Suction on an Oscillatory MHD Channel Flow with Heat Transfer." Applications \& Applied Mathematics 11, no. 1 (2016) 266-284.

[17] C. Johnson. "Numerical Solution of Partial Differential Equations by the Finite Element Method." Dover Publications: New York. 